\title{
Sequential Information Disclosure in Auctions
}

\author{
Dirk Bergemann ${ }^{\mathrm{a}}$, Achim Wambach ${ }^{\mathrm{b}}$ \\ ${ }^{a}$ Department of Economics, Yale University, New Haven, CT 06520, U.S.A. dirk.bergemann@yale.edu. \\ ${ }^{b}$ Department of Economics, University of Cologne, Cologne, Germany, wambach@wiso.uni-koeln.de.
}

\begin{abstract}
We propose a sequential auction mechanism for a single object in which the seller jointly determines the allocation and the disclosure policy. A sequential disclosure rule is shown to implement an ascending price auction in which each losing bidder learns his true valuation, but the winning bidder's information is truncated from below. As the auction ends, the winning bidder only has limited information, namely that his valuation is sufficiently high to win the auction. The sequential mechanism implements the allocation of the handicap auction of Eső and Szentes [10] but strengthens the participation constraints of the bidders from interim to posterior constraints. Due to the limited disclosure of information, the participation constraints (and incentive constraints) of all the bidders are satisfied with respect to all information revealed by the mechanism. In the special case in which the bidders have no private information initially, the seller can extract the entire surplus. Key words: Independent Private Value Auction, Sequential Disclosure, Ascending Auctions, Information Structure, Interim Equilibrium, Posterior Equilibrium.
\end{abstract}

\footnotetext{
ॠ We acknowledge financial support through NSF Grants SES 0851200 and ICES 1215808 and the German Research Foundation FOR 1371. We thank the Special Issue Editor, Alessandro Pavan, and the referees for many thoughtful comments. We are grateful to Vitali Gretschko and Xianwen Shi for many productive discussions. We thank Áron Tóbiás for excellent research assistance.
} 


\section{Introduction}

\subsection{Motivation}

We propose a sequential auction mechanisms for a single object and a finite number of bidders with independent private values. Importantly, the design of the mechanism encompasses the joint determination of the allocation of the object and the disclosure of the private information.

The present analysis is motivated by the observation that in many instances the seller of an object has considerable control over the information that the buyers have when bidding for the object under consideration. In fact, in some auctions, the seller intentionally limits the amount of information regarding the object sold to such an extent that they are commonly referred to as "blind auctions", see for example Kenney and Klein [16] and Blumenthal [4] for the licensing of motion pictures and Kavajecz and Keim [15] for trading of large asset portfolios.

Interestingly, the relevant information is frequently disclosed sequentially and systematically linked to the bidding mechanism. In an auction practice referred to as indicative bidding, the seller (or an agent of the seller) initially invites "indicative" bids on the basis of a prospectus with a limited description of the asset and subsequently grants access to additional and more precise information only on the basis of sufficiently strong interest as expressed in the early rounds of bidding, see Ye [21] or Boone and Goeree [5]. Similarly, in procurement auctions, in the "request for quote" process the buyer initially provides limited information about the project to the potential suppliers, which hand in a quote. On the basis of this first stage of the process, selected suppliers are invited who obtain further, more detailed information. In this procedure the improved specification of the project goes in parallel with negotiations of prices and conditions. The number of potential suppliers is reduced over time, until the winner is determined. Thus, in this sequential procedure suppliers learn more about the specification (and therefore about their costs) and only those able to compete further remain in the bidding process, see Beil and Wein [1].

Here, we shall investigate the nature of a sequential mechanism in which the seller can jointly determine the allocation and the disclosure rule. Importantly, we shall explicitly allow for sequential disclosure rules, i.e. disclosure rules which depend on the current (and past) bids, and hence in a direct mechanism on the current (and past) disclosed information. The sequential mechanism that we consider implements the allocation of the handicap auction of Eső and Szentes [10]. Beyond the implementation, the proposed mechanisms strengthens the participation constraints of the bidders from interim to posterior constraints. The sequential disclosure rule has the feature that each losing bidder learns his true valuation, but the winning bidder's information is truncated from 
below. As the auction ends, the winning bidder only has limited information, namely that his valuation is sufficiently high to win the auction. Due to the limited disclosure of information, the participation constraints (and the incentive constraints) of all the bidders are satisfied with respect to all information revealed by the mechanism, hence posterior constraints in the sense of Green and Laffont [13].

The interaction between the bidding and the disclosure process can be described within an ascending auction format, say in the form of the Japanese button auction, in which the asking price is raised continuously over time, see Cassady [8]. At each point in time and associated current price, each bidder has to make a decision as to whether he is staying in the auction or exiting the auction, i.e. whether he continues to press the button or whether he releases the button. Suppose for the moment, that initially each bidder would only know the common prior over his possible valuations. We may then ask how much additional information would a bidder minimally need to participate in an efficient bidding mechanism, i.e. a mechanism which would support the efficient allocation of the object across the bidders. Now, given a current price, all he would need to know is whether his value is above or below the current price. If indeed he were in the possession of this information at all past and hence lower price points, then the sequential disclosure policy that supports this information structure is simply that at price $p$ the true value $p$ is revealed. Thus as the current price increases, and a bidder learns his value, he will rationally drop out (at the next price point) and the remaining bidders are those who know that their true value is above the current price. This ascending auction terminates when all but one of the bidders have dropped out, and the remaining bidder is the winner of the auction. The associated assignment of the object is efficient as his value is larger than that of everybody else. Now, given the information that he has, his expected valuation is the conditional expectation of his value, given that it is larger than or equal to the current price $p$. In the canonical ascending auction he indeed would pay $p$, but given his current information, his willingness to pay is his conditional expectation, which is strictly larger than $p$.

From the point of view of the seller, she would like the bidders to have and hence to provide just enough private information to identify which bidder has the largest valuation. At the same time, she does not want to give the bidder with the largest valuation too much information on his valuation so as to minimize the informational rent of the winning bidder. In the above procedure, this is achieved by giving the bidder at each point in time a binary information partition by which each bidder learns whether his valuation is above or below some threshold. The subsequent game is such that if the valuation of the bidder lies below the threshold, it is optimal for him to exit the 
contest. Increasing the threshold for all bidders until only one bidder remains, and then charging the winning bidder his expected valuation, conditional on the valuation being larger than the final threshold, is the final outcome of the disclosure mechanism. Thus, each bidder learns either his true valuation, namely a losing valuation, or that he is the winning bidder and has the largest valuation, yet without learning its exact value.

If each bidder has private information, his type, from the very beginning of the auction, then the procedure needs to be generalized. First, the bidders have to report their types. Then, based on the reports, the thresholds in the sequential procedure are determined. These thresholds typically vary with the reports and hence differ across the bidders. Otherwise, the procedure works as above. Bidders obtain more and more information, and those who learn their true valuations exit the process. The final winner only learns that his valuation exceeds the final threshold. The winner will then be charged a price which is larger than this threshold but smaller than his expected value, conceding the informational rent he obtains with regard to his interim information. Determining the thresholds and the price is the critical step in the analysis to ensure that the bidder with the highest "shock-adjusted virtual valuation" wins, and to ensure that truthtelling is guaranteed, both with regard to the initial, that is interim information, and to the sequentially disclosed, that is posterior information.

The disclosure rule controls the informativeness of the private signal about the valuation. Importantly, while the seller determines the disclosure rule, the seller does not observe the realization of the private signal of each bidder. Formally, the disclosure rule is a mapping, one for each agent, from the value of the object to a distribution over a set of possible signals. The set of feasible disclosure rules includes the full disclosure rule, in which each agent learns his value perfectly, and the zero disclosure rule, in which each agent learns nothing above the common prior over the valuation. Between these two extreme disclosure rules are many other feasible disclosure rules, including deterministic and stochastic disclosure rules. ${ }^{1}$ The disclosure mechanism is subject to the standard incentive and participation constraints of the agents. Given the disclosed private information, each bidder has an incentive to report his private information truthfully, and given the private informa-

\footnotetext{
${ }^{1}$ Kamenica and Gentzkow [14] consider a related class of problems referred to as "Bayesian Persuasion". They consider the interaction between a principal and a single agent, where the principal can determine the disclosure rule, but the allocation is determined by the agent. Thus the game is "given" rather than "designed" as in the current analysis, but of course the action taken by the agent can be influenced through the disclosure rule adopted by the principal.
} 
tion, each bidder is willing to participate, i.e. his expected net utility is at least as large as his utility from not participating. We shall refer to these constraints as the posterior incentive and posterior participation constraints, as each agent is conditioning his report and his participation on the private information revealed in the disclosure mechanism. These notions of posterior constraints were first introduced by Green and Laffont [13] to reflect the possibility that the mechanism may reveal some, but not necessarily all, payoff-relevant information to the agents. ${ }^{2}$

\subsection{Related Literature}

Bergemann and Pesendorfer [2] consider the standard independent private value auction for a single good. In a static mechanism design problem, the seller jointly determines the allocation and the disclosure rule of the mechanism, and the design is subject to the posterior incentive and posterior participation constraints. The disclosure rule of the mechanism determines the informativeness of the private signal that each agent receives about his true value for the object. The optimal disclosure mechanism uses a deterministic, but coarse, disclosure rule. Each agent receives only limited information about his true value, and the resulting revenue strictly exceeds the revenue of the full disclosure rule. The optimality of the coarse information arises from the nature of the information rent. In the complete disclosure rule, each agent learns his true value, and while this guarantees an efficient allocation, it allows the agent to receive a substantial information rent. By limiting the private information, the seller can reduce the information rent without a substantial decrease in the efficiency of the allocation.

Gershkov [11] reconsiders the optimal disclosure mechanism of Bergemann and Pesendorfer [2] under a weaker participation constraint, namely the ex-ante participation constraint, while maintaining the posterior incentive constraints. With the ex-ante participation constraint, the seller can charge each bidder a participation fee before the release of any private information and extract the entire expected surplus from the agents. ${ }^{3}$

In an important contribution, Eső and Szentes [10] pursue the analysis of the optimal information disclosure in the context of an informational environment which encompasses Bergemann and

\footnotetext{
${ }^{2}$ By contrast, the ex-post incentive and participation constraints are evaluated under complete information about the realized valuation of each agent, thus ex post. By convention, we refer to ex ante as the moment at which the bidders only know the common prior, and to interim as the moment at which each bidder knows his own private type.

${ }^{3}$ The nature of the solution in Gershkov [11] is reminiscent to the analysis of the efficient regulation of a natural monopoly offered by Demsetz [9] and Loeb and Magat [19], which suggests the ex ante sale of all future rents.
} 
Pesendorfer [2] and Gershkov [11]. In their model, each agent has two possible sources of private information, an initial estimate of the true value of the object, the type, and subsequently a signal that informs him about the realization of his true value. Eső and Szentes [10] show that the additional, or incremental information relative to the initial estimate, can be represented as a signal that is orthogonal to, i.e. independent of, the type. Based on this representation of the private information of each agent, namely the initial signal and the incremental and independent signal, they suggest a sequential screening contract, in which each agent first reveals his initial information, and then in a second step the additionally disclosed information. ${ }^{4}$ Importantly, the disclosure of the initial estimate, the type, cannot be affected by the disclosure mechanism, it is only the disclosure of the subsequent, orthogonal signal that is controlled by the disclosure mechanism. The design of the optimal disclosure mechanism is subject to the posterior incentive constraints, but only the interim participation constraints. In particular, the mechanism requires each bidder to pay a participation fee, which modifies the probability of winning, and a transfer conditional on winning. Thus the mechanism necessitates a payment from the losing bidders, and hence violates the posterior participation constraints. Thus, this result leaves open the question what can be achieved under stronger participation constraints.

Surprisingly, Eső and Szentes [10] show that the optimal disclosure mechanism is the full disclosure mechanism, and show that the optimal disclosure mechanism generates as much revenue as an optimal mechanism could in which the incremental information of each agent was observable by the seller. ${ }^{5}$ In a recent contribution, Li and Shi [18] extend the analysis of the optimal static disclosure mechanism by permitting the disclosure process to depend on the true value of the object, but not on the orthogonal signal. In this case, they show that the optimal policy can involve partial and discriminatory rather than complete and uniform information disclosure. The design of dynamically optimal disclosure rules is also analyzed in sequential contracting problems such as in auctions with resale or in vertical relationships, see e.g. Calzolari and Pavan [6], [7], and Lebrun [17]. In these environments, the information regarding the current transaction influences the distribution of the surplus in future transactions, and over the time the identity of the trading partner changes.

\footnotetext{
${ }^{4}$ The decomposition between the initial and the incremental signal proved, by itself, to be an important tool in the analysis of sequential screening contracts, see Pavan, Segal, and Toikka [20] for a recent contribution on revenue maximizing mechanism design in a general environment with an infinite time horizon.

${ }^{5}$ Gershkov [12] obtains a similar result in a setting where the incremental signal of each agents pertains to common value component in the valuation of each bidder.
} 
And while the resulting disclosure policy is still primarily driven by the concern for the information rents, the trade-offs are driven by more subtle considerations regarding the incidence of rents over time.

We proceed as follows. In the next section we present the model and describe the sequential disclosure mechanism. In Section 3 we analyze the case without interim private information by the bidders; and here the first best allocation can be implemented. The general case is analyzed in Section 4 . Section 5 concludes with a discussion of possible extensions and applications.

\section{The Model}

\subsection{Payoffs, Types and Signals}

There is one seller with a single object for sale and there are $n$ potential bidders, indexed by $i \in\{1,2, \ldots, n\}$, which are risk-neutral and with quasi-linear utility. The seller can commit to a mechanism to sell the object to one of the competing bidders.

The true valuation of bidder $i$ is given by $V_{i} \in \mathcal{V}_{i}$, where $\mathcal{V}_{i}$ is a subset of $\mathbb{R}_{+}$, which we assume without loss of generality to be equal to the unit interval $\mathcal{V}_{i}=(0,1]$ for all $i$. The prior distribution of $V_{i}$ is denoted by $H_{i}$ and corresponding density $h_{i}$. The valuations are independently distributed across the agents. The cost $c$ of producing the object for the seller is normalized without loss of generality to 0 .

Each agent receives a (noisy) signal $v_{i}$ of his true valuation $V_{i}$ before he enters the mechanism. We assume that the type $v_{i}$ is distributed, again without loss of generality on the unit interval $[0,1]$ with distribution $F_{i}$ and corresponding density $f_{i}$. We denote by $H_{i v_{i}}\left(V_{i}\right) \triangleq H_{i}\left(V_{i} \mid v_{i}\right)$, the distribution of $V_{i}$ conditional on $v_{i}$, with the corresponding conditional density $h_{i v_{i}}\left(V_{i}\right) \triangleq h_{i}\left(V_{i} \mid v_{i}\right)$. We refer to $v_{i}$ as the type, or interim information, of agent $i$.

In addition, each agent $i$ may receive additional information which resolves the residual uncertainty about the value $V_{i}$ during the bidding process. We describe the additional information by a random variable $s_{i} \in S_{i}=(0,1]$ and refer to it as signal $s_{i}$ with a given conditional distribution $G_{i v_{i}}\left(s_{i}\right) \triangleq G_{i}\left(s_{i} \mid v_{i}\right)$. By observing the signal $s_{i}$ (together with type $v_{i}$ ) the bidder learns his true valuation $V_{i}$, or

$$
V_{i} \triangleq u_{i}\left(v_{i}, s_{i}\right)
$$

\subsection{Sequential Mechanism}

We consider a specific sequential disclosure and allocation mechanism that ends with the allocation of the object. The mechanism itself is an indirect mechanism that embeds the disclosure 
process in an ascending auction. The indirect mechanism is specifically tailored to implement the allocation of the handicap auction of Esö and Szentes [10] with posterior participation constraints. As such, it does not attempt to find the revenue maximizing mechanism for all possible disclosure policies, and in particular, it does not attempt to provide a dynamic counterpart to the static analysis of Li and Shi [18].

The disclosure component of the mechanism determines the time by which the signal $s_{i}$ is revealed. The allocation component determines the final allocation of and payments for the object. As in the ascending auction, the object is awarded to the final active bidder. Importantly, the seller can commit to a disclosure mechanism that determines when and how the information contained in the signal $s_{i}$ is disclosed to bidder $i$. And while the seller determines the disclosure mechanism, the realized information remains private information to each bidder $i$. The specific disclosure of the random variable $s_{i}$ is sequential in that the disclosure mechanism determines for every realization of the signal $s_{i}$ the time at which the realization is disclosed. In particular, higher realizations of $s_{i}$ are going to be disclosed later in time.

Disclosure. The sequential mechanism asks each bidder to initially report his type $v_{i}$ and then to report his signal realization $s_{i}$ as soon as it is disclosed by the mechanism. The disclosure part of the mechanism determines the time $t \in[0,1]$ at which the signal realization $s_{i}$ is disclosed. We first define the sequential disclosure component which determines the time at which the signal realization $s_{i}$ is disclosed. For every agent $i$, we define a disclosure function $\xi_{i} \triangleq \xi_{i}\left(t, \widehat{v}_{i}, s_{i}\right)$,

$$
\xi_{i}:[0,1] \times[0,1] \times(0,1] \rightarrow[0,1],
$$

which determines the disclosure of the signal realization as a function of time $t \in[0,1]$, reported type $\widehat{v}_{i} \in[0,1]$ and signal realization $s_{i} \in(0,1]$. The disclosure function $\xi_{i}$ is assumed to be a step function in time $t$, with a single jump, from 0 (which represents the event of no signal disclosure yet) to $s_{i}>0$ at a particular disclosure time $t_{i}\left(\widehat{v}_{i}, s_{i}\right)$,

$$
t_{i}\left(\widehat{v}_{i}, s_{i}\right) \triangleq \min \left\{t \in[0,1] \mid \xi_{i}\left(t, \widehat{v}_{i}, s_{i}\right)>0\right\}
$$

and constant everywhere else in $t$. Thus the disclosure time $t_{i}\left(\widehat{v}_{i}, s_{i}\right)$ is the time at which the signal realization $s_{i}$ is disclosed to bidder $i$ given a reported type $\widehat{v}_{i}$. The state of the disclosure process at time $t$, given by $\xi_{i}\left(t, \widehat{v}_{i}, s_{i}\right)$, is privately observed by bidder $i$, and it is either 0 (which means 
disclosure has not yet occurred) or $s_{i}$ (which means disclosure has occurred). ${ }^{6}$

Reporting Strategy. A reporting (or message) strategy $m_{i}=\left(r_{i}, d_{i}\right)$ of bidder $i$ consists of an initial report $r_{i}$ and a (continued) participation decision $d_{i}$ for bidder $i$. The strategy of each bidder $i$ depends on the private state (or history) of bidder $i$. The private history of bidder $i$ at $t=0$ is simply his type $v_{i}$, or $h_{i}^{0}=\left(v_{i}\right)$ and at all subsequent times $t>0$, his type $v_{i}$, his reported type $\widehat{v}_{i}$ and the state of the disclosure process $\xi_{i}\left(t, \widehat{v}_{i}, s_{i}\right)$, thus $h_{i}^{t}=\left(v_{i}, \widehat{v}_{i}, \xi_{i}\left(t, \widehat{v}_{i}, s_{i}\right)\right)$. Formally, then the initial report $r_{i} \triangleq r_{i}\left(v_{i}\right)$ is defined as a mapping,

$$
r_{i}:[0,1] \rightarrow[0,1]
$$

and the participation (or continuation) decision $d_{i} \triangleq d_{i}\left(t, v_{i}, \widehat{v}_{i}, \xi_{i}\left(t, v_{i}, s_{i}\right)\right)$ with

$$
d_{i}:[0,1] \times[0,1] \times[0,1] \times[0,1] \rightarrow\{0,1\}
$$

The decision of the bidder is either to stay in the bidding process: $d_{i}(\cdot)=1$ or to exit the bidding process: $d_{i}(\cdot)=0$. The participation decision depends on the time $t \in[0,1]$, the true type $v_{i}$, the reported type $\widehat{v}_{i} \in[0,1]$, and the state of the disclosure process $\xi_{i}\left(t, \widehat{v}_{i}, s_{i}\right) \in[0,1]$. The exit decision is irrevocable, and hence $d_{i}$, as a function of time, is restricted to be weakly decreasing in $t$.

Allocation. The object is assigned as soon as all but one of the bidders have exited the bidding process. As time $t$ progresses, we can track the exit decision of the agents. At time $t<1$, agent $i$ has exited the bidding process if the exit time $\tau_{i}(t)$ of bidder $i$,

$$
\tau_{i}(t) \triangleq \min \left\{\left\{t^{\prime} \leq t \mid d_{i}\left(t^{\prime}, \cdot\right)=0\right\} \wedge 1\right\}
$$

satisfies $\tau_{i}(t) \leq t$. To wit, if the agent has not yet exited, then at time $t$, he is assigned the exit time 1, which simply represents the fact that at $t$ he is still active. For bidder $i$, the disclosure process $\xi_{i}(\cdot)$ stops as soon as he exits the auction, or $\xi_{i}\left(t, \widehat{v}_{i}, s_{i}\right)=\xi_{i}\left(\tau_{i}, \widehat{v}_{i}, s_{i}\right)$ for all $t \geq \tau_{i}$.

The mechanism determines the allocation at the first time, $\tau$, at which all but one of the agents have exited the auction:

$$
\tau \triangleq \min \left\{t>0 \mid \exists k, \text { s. th. } \tau_{j}(t) \leq t, \forall j \neq k, \tau_{k}(t)>t\right\}
$$

\footnotetext{
${ }^{6}$ We restrict $s_{i}$ and $V_{i}$ to the half-open interval $(0,1]$ (rather than the closed interval $[0,1]$ ) for the sole purpose of identifying the report $\xi_{i}(\cdot)=0$ with the event of no signal disclosure yet.
} 
This definition of the stopping time (and the subsequent definition of the allocation rule) excludes events in which all of the remaining bidders stop at the same time. ${ }^{7}$

The assignment of the object is described by a probability vector $x=\left(x_{1}, \ldots, x_{n}\right)$, and the assignment probabilities $x_{i}$,

$$
x_{i}:[0,1] \times[0,1] \rightarrow\{0,1\}
$$

are required to sum to less than or equal to one. The allocation itself depends only on the exit time $\tau_{i}$ of bidder $i$ and the stopping time $\tau$ of the auction, i.e.

$$
x_{i}\left(\tau_{i}, \tau\right) \triangleq 0 \Leftrightarrow \tau_{i} \leq \tau, \quad x_{i}\left(\tau_{i}, \tau\right) \triangleq 1 \Leftrightarrow \tau_{i}>\tau
$$

Similarly, the transfers are described by a vector $p=\left(p_{1}, \ldots, p_{n}\right)$, where each $p_{i}$ is determined by a mapping:

$$
p_{i}:[0,1] \times[0,1] \times[0,1] \rightarrow \mathbb{R}_{+}
$$

The transfer payments will have the property that only the winning bidder is making a positive payment, i.e. $p_{i}\left(\widehat{v}_{i}, \tau_{i}, \tau\right)=0$ if $\tau_{i} \leq \tau$, and that the payment of the winning bidder will only depend on his initial report $\widehat{v}_{i} \in[0,1]$, and the stopping time $\tau \in[0,1]$.

Incentive and Participation Constraints. We define "truthtelling" for agent $i, m_{i}^{*}=\left(r_{i}^{*}, d_{i}^{*}\right)$ by

$$
r_{i}^{*}\left(v_{i}\right) \triangleq v_{i}
$$

and

$$
d_{i}^{*}\left(t, v_{i}, v_{i}, \xi_{i}\left(t, v_{i}, s_{i}\right)\right) \triangleq \begin{cases}1, & \text { if } \xi_{i}=0 \\ 0, & \text { if } \xi_{i}>0\end{cases}
$$

In other words, each agent reports truthfully his own type, and then stays in the bidding process as long as he has not yet received the additional signal $s_{i}$, and exits as soon as a signal has been received. We refer to this as "truthtelling" behavior as the individual exit time reveals the value of the signal.

In the sequential mechanism, we determined the allocation $x_{i}$ and the payment $p_{i}$ in terms of the exit time $\tau_{i}$, the stopping time $\tau$, and the reported type $\widehat{v}_{i},(5)$ and (6) respectively. Now, the exit time and the stopping time are induced by the reporting strategies of all the players, and to

\footnotetext{
${ }^{7}$ These are zero probability events and hence can be omitted without loss of generality. At the expense of additional notation, we could complete the description by introducing a uniform random allocation in case of such a zero probability event, essentially a tied bid.
} 
make this dependence explicit we can express allocations and transfer payments directly in terms of the message profile $m=\left(m_{i}, m_{-i}\right)$ in the obvious way:

$$
X_{i}\left(m_{i}, m_{-i}\right) \triangleq x_{i}\left(\tau_{i}\left(m_{i}\right), \tau(m)\right), \quad P_{i}\left(m_{i}, m_{-i}\right) \triangleq p_{i}\left(v_{i}\left(m_{i}\right), \tau_{i}\left(m_{i}\right), \tau(m)\right) .
$$

We can now define the posterior incentive and participation constraints. We require that truthtelling satisfies the incentive constraints along every private history $h_{i}^{t}$ (consistent with the mechanism).

$$
\mathbb{E}\left[X_{i}\left(m_{i}^{*}, m_{-i}^{*}\right) V_{i}-P_{i}\left(m_{i}^{*}, m_{-i}^{*}\right) \mid h_{i}^{t}\right] \geq \mathbb{E}\left[X_{i}\left(m_{i}, m_{-i}^{*}\right) V_{i}-P_{i}\left(m_{i}, m_{-i}^{*}\right) \mid h_{i}^{t}\right], \forall m_{i}, \forall h_{i}^{t},
$$

and that truthtelling satisfies the participation constraints along every private history $h_{i}^{t}$ (consistent with the mechanism):

$$
\mathbb{E}\left[X_{i}\left(m_{i}^{*}, m_{-i}^{*}\right) V_{i}-P_{i}\left(m_{i}^{*}, m_{-i}^{*}\right) \mid h_{i}^{t}\right] \geq 0, \forall h_{i}^{t}
$$

The incentive constraints given by (8) thus cover the reporting behavior of each agent for the entire history of the mechanism. But, of course the reporting is subject to the rules of the mechanism, namely the initial report $\widehat{v}_{i}$ and any exit decision during the disclosure process are irrevocable. In particular, the above incentive and participation constraints imply that the initial set of constraints, the interim, and the terminal participation and incentive, the posterior constraints are satisfied; namely at $t=0$ when each agent only observes his type $v_{i}: h_{i}^{0}=v_{i}$, and at $t=\tau$ when the mechanism terminates with the allocation of the object. In fact, the notion of posterior implementation evaluates the constraints at all private histories (information sets) that can be reached in the mechanism. Thus the set of constraints are determined by the mechanism itself, and in this sense "endogenous" to mechanism. To the extent then that the mechanism does not reveal all possible information about the true willingness to pay of the bidders, as it will typically be the case, the constraints are weaker than the ex-post constraints which would apply if all the private information had become public. The subsequent results of the posterior implementation, Proposition 1 and 2 , establish that the participation constraints can be substantially strengthened if the constraints are "measurable" with respect to all disclosed information, but not beyond that. In the present context of the optimal auction, it means that the seller does not have to use participation payments, but rather can rely exclusively on transaction payments. In other words, the commitment power of the bidders can be substantially weakened in the sense that the commitment of bidder is only required at the time of the assignment of the object rather at the very beginning of the bidding process. 
Summary of Sequential Mechanism. We summarize the sequential mechanism as follows. For each bidder $i$, nature initially draws $\left(v_{i}, s_{i}\right)$. Bidder $i$ initially observes $v_{i}$ but not $s_{i}$. Bidder $i$ reports $\widehat{v}_{i} \triangleq r_{i}\left(v_{i}\right)$ according to the reporting strategy $r_{i}(\cdot)$ (whether or not $\widehat{v}_{i}=v_{i}$ ). Then, the disclosure policy $\xi_{i}(\cdot)$ uses the reported type $\widehat{v}_{i}$ (and not the true type $v_{i}$ ) and the signal $s_{i}$ to generate the disclosure time $t\left(\widehat{v}_{i}, s_{i}\right)$. At any point of time $t$, the bidder either knows that $s_{i}>s_{i}^{\prime}$ for the critical signal $s_{i}^{\prime}$ such that $t=t\left(\widehat{v}_{i}, s_{i}^{\prime}\right)$ or that the value is $s_{i}$, namely if $t\left(\widehat{v}_{i}, s_{i}\right) \leq t$.

The allocation mechanism is thus a version of an ascending auction, in the format of the "Japanese" or "button" auction in which the price uniformly increases over time. In the button auction, if a bidder releases the button, he reveals his type, and the auction ends for him. The ascending disclosure mechanism modifies the button auction in two important aspects: $(i)$ it associates a disclosure process with the price process, $(i i)$ the final price paid is personalized, and related to, but not necessarily equal to the valuation of the final remaining competitor.

\section{Bidding without Interim Information}

We begin our analysis with bidders who do not possess interim private information. In other words, the initial information of each agent is simply the common prior $H=\left(H_{i}\right)_{i=1}^{n}$ over the valuations $V=\left(V_{i}\right)_{i=1}^{n}$. This informational environment with uninformed bidders was analyzed by Bergemann and Pesendorfer [2], but they restricted attention to static disclosure mechanisms. In this section we revisit their setting but allow for the possibility of sequential information disclosure. The purpose of this section is to present a simple and hopefully transparent environment to understand how information disclosure, efficiency, and revenue extraction are naturally linked in the ascending mechanism.

We now adapt (and simplify) the sequential mechanism, defined earlier by (1), (5) and (6) to the present environment. In particular, without interim information $v_{i}$, the disclosure function can depend on time $t$ and signal $s_{i}$ alone, and without loss of generality, we take the signal $s_{i}$ to be equal to the valuation $V_{i}$. With this, the disclosure function $\xi_{i}$ is given by

$$
\xi_{i}:[0,1] \times[0,1] \rightarrow[0,1]
$$

which determines the disclosure of the valuation as a function of time $t \in[0,1]$ and of the valuation $V_{i} \in[0,1]$.

In the absence of interim private information, we can choose the disclosure functions $\left\{\xi_{i}\right\}_{i=1}^{n}$ to 
be identical for all of the agents and define

$$
\xi_{i}\left(t, V_{i}\right) \triangleq\left\{\begin{array}{cl}
0, & \text { if } t<V_{i} \\
V_{i}, & \text { if } t \geq V_{i}
\end{array}\right.
$$

Thus, bidder $i$ with valuation $V_{i}$ receives a perfectly informative signal about his valuation at $t=V_{i}$, whereas at all times $t$ with $t<V_{i}$, he will infer that his expected valuation is given by the conditional expectation, $\mathbb{E}\left[V_{i} \mid V_{i} \geq t\right]$.

The assignment of the object to agent $i$ depends only on his exit time $\tau_{i}$ and the stopping time $\tau$,

$$
x_{i}\left(\tau_{i}, \tau\right) \triangleq\left\{\begin{array}{lll}
0, & \text { if } & \tau_{i} \leq \tau \\
1, & \text { if } & \tau_{i}>\tau .
\end{array}\right.
$$

The transfer payments request a single positive payment $p_{i}$ at the stopping time $\tau$ from the winning bidder only:

$$
p_{i}\left(\tau_{i}, \tau\right) \triangleq\left\{\begin{array}{ccc}
0, & \text { if } & \tau_{i} \leq \tau \\
\mathbb{E}\left[V_{i} \mid V_{i} \geq \tau\right], & \text { if } & \tau_{i}>\tau .
\end{array}\right.
$$

A sequential mechanism is then defined by (11)-(13), and we shall refer to it as the ascending disclosure mechanism.

It is then optimal for the bidder to stay in the bidding process if no information has been revealed: $\xi_{i}(t, \cdot)=0$; and to exit if information has been disclosed: $\xi_{i}(t, \cdot)=V_{i}$. We can now state our first result in the setting with bidders without interim information.

Proposition 1. The ascending disclosure mechanism satisfies the posterior incentive and participation constraints for all agents and the seller extracts the entire social surplus.

Proof. We first observe that if all the bidders follow the truthtelling strategy, then the posterior participation constraint is satisfied for the losing and the winning bidders. A losing bidder does not receive the object, see allocation rule (12), and by the payment rule (13) faces a zero payment, and hence his net utility is equal to zero. The winning bidder receives the object with probability one, see allocation rule (12), but given the payment rule (13) has to pay his expected conditional valuation at the stopping time $\tau$. Thus, again, given the information disclosed by the mechanism at time $\tau$, the net utility of the winning bidder is zero, and hence the posterior participation constraint is satisfied.

We then consider the posterior incentive constraints in the ascending disclosure mechanism. Every losing bidder learns his value and immediately exits to receive a net utility of zero. Clearly, 
exiting before learning the valuation $V_{i}$ does not improve the net utility of bidder $i$, as bidder $i$ would merely exit earlier, and still receive zero net utility. But if he were to stay longer, and not stop his own disclosure process, then the auction could reach the stopping point $\tau>\tau_{i}=V_{i}$, and ask bidder $i$ to pay more than his true valuation. Clearly, this does not improve his net utility either. Finally, consider the winning bidder. He cannot change the price conditional on winning, he can only lower his probability of winning by exiting the auction before his valuation is revealed. But if he were to exit the auction, he would receive zero net utility as well, thus exiting early does not constitute a profitable deviation either. Thus staying in the mechanism is an optimal strategy.

Finally, let us consider the revenue of the ascending disclosure mechanism. The seller receives revenue from bidder $i$ when all the other bidders have a valuation below him. Thus, the allocation is efficient, and as every bidder, winning or losing receive zero expected utility, it follows that the seller receives the entire social surplus.

We observe that in the ascending disclosure mechanism, the participation and incentive constraints of the losing bidders are not merely satisfied as posterior constraints, but even hold as ex post constraints. In other words, given the truthful reports of all the agents, a losing bidder would not want to change his reporting behavior, even after he learned his true valuation $V_{i}$. In contrast, for the winning bidder, the surplus extraction result crucially relies on the fact that the winning bidder does not learn his true valuation $V_{i}$, but rather is limited to knowing that his true valuation is in the interval $[\tau, 1]$ and hence forms his conditional expectation on the basis of the disclosed information.

Having shown that with ex-ante uninformed bidders, the ascending information disclosure leads to the revenue maximizing allocation, we now generalize the procedure to the case where the bidders have some private, or interim, information before they enter the mechanism.

\section{Bidding with Interim Information}

We now turn to the general model in which each bidder $i$ receives a noisy signal $v_{i}$ of his valuation $V_{i}$, his interim information. We provide a sequential implementation of the static mechanism in Eső and Szentes [10] that differs in two essential aspects from their implementation: $(i)$ the signal $s_{i}$ is not completely disclosed, and $(i i)$ the participation constraint of each bidder is satisfied at the posterior level rather than merely at the interim level.

We maintain the informational environment in Eső and Szentes [10], namely that the density $f_{i}\left(v_{i}\right)$ associated with the distribution $F_{i}\left(v_{i}\right)$ of the buyer's type $v_{i}$ is positive everywhere and 
that the distribution satisfies the monotone hazard condition, that is $f_{i}\left(v_{i}\right) /\left(1-F_{i}\left(v_{i}\right)\right)$ is weakly increasing in $v_{i}$. In addition, the relationship between the initial type and final valuation, namely $\left(\partial H_{i v_{i}}\left(V_{i}\right) / \partial v_{i}\right) / h_{i v_{i}}\left(V_{i}\right)$ is assumed to be increasing in $v_{i}$ and $V_{i}$. Finally, we adopt their orthogonal representation that the signal $s_{i}$ simply represents the percentile of the true valuation and thus write $s_{i}$ as

$$
s_{i} \triangleq s_{i}\left(v_{i}, V_{i}\right)=H_{i v_{i}}\left(V_{i}\right)
$$

As $s_{i}$ is the percentile of the true valuation conditional on the type $v_{i}$, the distribution $G_{i v_{i}}\left(s_{i}\right)$ of $s_{i}$ conditional on $v_{i}$ is simply the uniform distribution on $[0,1]$ for all $v_{i}$.

We proceed in three steps. In Subsection 4.1, we recall the relevant aspects of the revenue maximizing allocation in which the signal profile $\mathbf{s}$ is directly observable by the seller, as derived by Eső and Szentes [10]. In Subsection 4.2, we present the ascending disclosure mechanism with interim information. In Subsection 4.3, we show that the ascending disclosure mechanism implements the revenue maximizing allocation with posterior incentive and participation constraints.

$A$ Caveat: Disclosure Contingent on $s_{i}$ versus $V_{i}$. We represent the additional information about $V_{i}$ contained in the signal $s_{i}$ relative to type $v_{i}$ by means of an orthogonal random variable as first suggested by Eső and Szentes [10]. And like them, we restrict the disclosure policy of the seller to use information about the signal $s_{i}$ only. We should emphasize that the representation of the additional information in form of an orthogonal signal is indeed without loss of generality. By contrast, the requirement that the disclosure policy is contingent on the reported type $\widehat{v}_{i}$ and the signal $s_{i}$ only (as in (1)), rather than on the true value $V_{i}$ is a substantial restriction. In a recent paper, Li and Shi [18] consider static disclosure policies in which the disclosure policy of the seller is allowed to use information about the value $V_{i}$ itself rather than $s_{i}$ (and the reported type $\widehat{v}_{i}$ ) only. In particular, they give an example, their Example 4, in which the disclosure policy based on the true value $V_{i}$ strictly dominates any disclosure policy based on $s_{i}$ alone. However we believe that the present arguments regarding the benefits of sequential relative to static disclosure mechanisms remain valid after allowing for policies contingent on $V_{i}$ rather than $s_{i}$. We shall detail our view at the end of Section 5 .

\subsection{Observable Signal}

The benchmark case is the situation where the seller can observe the signal $s_{i}$ of each bidder. Eső and Szentes [10] show that in the second best, where the seller can observe the so-called 'shocks' $s_{i}$, the optimal mechanism has the following property: the object is rewarded to the bidder with 
the largest non-negative "shock adjusted virtual valuation" $W_{i}\left(v_{i}, s_{i}\right)$,

$$
W_{i}\left(v_{i}, s_{i}\right)=u_{i}\left(v_{i}, s_{i}\right)-\frac{1-F_{i}\left(v_{i}\right)}{f_{i}\left(v_{i}\right)} u_{i 1}\left(v_{i}, s_{i}\right),
$$

where $u_{i 1}\left(v_{i}, s_{i}\right)$ is the partial derivative of $u_{i}\left(v_{i}, s_{i}\right)$ with respect to $v_{i}$, thus the impulse response of $u_{i}$ with respect to $v_{i}$. We next describe some properties of the virtual valuation. ${ }^{8}$

\section{Lemma 1 (Virtual Valuation).}

1. The virtual valuation $W_{i}\left(v_{i}, s_{i}\right)$ is strictly increasing in $v_{i}$ and $s_{i}$;

2. If $u_{i}\left(v_{i}, s_{i}\right)=u_{i}\left(v_{i}^{\prime}, s_{i}^{\prime}\right)$ and $v_{i} \geq v_{i}^{\prime}$, then $W_{i}\left(v_{i}, s_{i}\right) \geq W_{i}\left(v_{i}^{\prime}, s_{i}^{\prime}\right)$;

3. If $W_{i}\left(v_{i}, s_{i}\right)=W_{i}\left(v_{i}^{\prime}, s_{i}^{\prime}\right)$ and $v_{i} \geq v_{i}^{\prime}$, then $u_{i}\left(v_{i}, s_{i}\right) \leq u_{i}\left(v_{i}^{\prime}, s_{i}^{\prime}\right)$.

Proof. (1.)-(3.) follow directly from Lemma 1 and Corollary 1 of Eső and Szentes [10].

The monotonicity of the virtual utility $W_{i}\left(v_{i}, s_{i}\right)$ implies that for a given vector of types $\mathbf{v}=$ $\left(v_{1}, \ldots, v_{n}\right)$ and vector of signals $\mathbf{s}_{-i}=\left(s_{1}, . ., s_{i-1}, s_{i+1}, \ldots, s_{n}\right)$, bidder $i$ obtains the good whenever his signal $s_{i}$ is larger than a threshold value $\underline{s_{i}}\left(\mathbf{v}, \mathbf{s}_{-i}\right)$ of the signal $s_{i}$. This threshold is defined as

$$
\underline{s_{i}}\left(\mathbf{v}, \mathbf{s}_{-i}\right) \triangleq \min \left\{\min \left\{s_{i} \in[0,1] \mid W_{i}\left(v_{i}, s_{i}\right) \geq 0 \text { and } \forall j \neq i, W_{i}\left(v_{i}, s_{i}\right) \geq W_{j}\left(v_{j}, s_{j}\right)\right\}, 1\right\} .
$$

Above we take the minimum over $s_{i}$ and 1 , as $v_{i}$ might be small, and hence there might be no signal $s_{i} \in[0,1]$ that would turn bidder $i$ into a winner. Given that the virtual valuation only depends on $\mathbf{v}$ and $\mathbf{s}$ and in particular is not a function of the distributional property of $\mathbf{s}$, we can construct the optimal (static) mechanism for every realization of $\mathbf{s}$. The optimal allocation is then determined by the virtual valuations and the bidder obtains the good whenever his type is larger than the threshold $\underline{v_{i}}\left(\mathbf{v}_{-i}, \mathbf{s}\right)$,

$$
\underline{v_{i}}\left(\mathbf{v}_{-i}, \mathbf{s}\right) \triangleq \min \left\{\min \left\{v_{i} \in[0,1] \mid W_{i}\left(v_{i}, s_{i}\right) \geq 0 \text { and } \forall j \neq i, W_{i}\left(v_{i}, s_{i}\right) \geq W_{j}\left(v_{j}, s_{j}\right)\right\}, 1\right\} .
$$

We construct incentive compatible transfers, which are only paid in case of winning, by asking the winner to pay the valuation of the lowest type $\underline{v_{i}}\left(\mathbf{v}_{-i}, \mathbf{s}\right)$, given the signals $\mathbf{s}$, which would have won the contest,

$$
p_{i}\left(\mathbf{v}_{-i}, \mathbf{s}\right) \triangleq u_{i}\left(\underline{v_{i}}\left(\mathbf{v}_{-i}, \mathbf{s}\right), s_{i}\right) .
$$

The payment $p_{i}\left(\mathbf{v}_{-i}, \mathbf{s}\right)$ therefore has the Vickrey property that the payment of winner $i$ is independent of his true type $v_{i}$, conditional on the event $v_{i} \geq \underline{v}_{i}\left(\mathbf{v}_{-i}, \mathbf{s}\right)$. Thus, the payment rule described by (18) implements truthtelling with respect to $v_{i}$ if the signals $\left(s_{1}, \ldots, s_{n}\right)$ are publicly revealed.

\footnotetext{
${ }^{8}$ If the seller has a strictly positive cost $c$ of providing the good, then the object is assigned if and only if the largest shock-adjusted virtual valuation is larger than $c$, and no further changes are necessary.
} 


\subsection{Ascending Disclosure Mechanism}

We now turn to the case where the additional signal $s$ is unobservable to the seller. We next construct the sequential information disclosure with the important property that the virtual valuations of all active bidders are equalized at all times $t$ until bidding ends at $\tau$. Given the initial reports of all bidders, truthful or not, we reveal to each bidder $i$ whether his signal $s_{i}$ is above a current threshold at a speed such that at all times the virtual utility of all active bidders evaluated at the current threshold are identical. In this context, the initial report $\widehat{v}_{i}$ of bidder $i$ simply determines the speed at which the disclosure process is running through the signals. Formally, we explicitly define the disclosure function $\xi_{i}\left(t, \widehat{v}_{i}, s_{i}\right)$ through the virtual valuation $W_{i}\left(\widehat{v}_{i}, s_{i}\right)$ and the associated disclosure time $t_{i}\left(\widehat{v}_{i}, s_{i}\right)$ for all $i, \widehat{v}_{i}, s_{i}$,

$$
t_{i}\left(\widehat{v}_{i}, s_{i}\right) \triangleq\left\{\begin{array}{ccc}
0, & \text { if } \quad W_{i}\left(\widehat{v}_{i}, s_{i}\right)<0 ; \\
W_{i}\left(\widehat{v}_{i}, s_{i}\right), & \text { if } \quad W_{i}\left(\widehat{v}_{i}, s_{i}\right) \geq 0 ;
\end{array}\right.
$$

and thus

$$
\xi_{i}\left(t, \widehat{v}_{i}, s_{i}\right)= \begin{cases}0, & \text { if } t<t_{i}\left(\widehat{v}_{i}, s_{i}\right) ; \\ s_{i}, & \text { if } t \geq t_{i}\left(\widehat{v}_{i}, s_{i}\right) .\end{cases}
$$

The disclosure time $t_{i}\left(\widehat{v}_{i}, s_{i}\right)$ is thus strictly increasing in both the reported type $\widehat{v}_{i}$ and the signal realization $s_{i}$. Thus, a higher reported type slows down the disclosure of information, and a higher realizations of $s_{i}$ is going to be disclosed later than a low realization of $s_{i}$. In this sense, the initial report $\widehat{v}_{i}$ influences the speed of disclosure, and as time goes by, the bidder continues to update his estimate, even in the absence of a disclosed signal. The disclosure function $\xi_{i}$ and disclosure time $t_{i}$ for different realization of the type $v_{i}$ and signal $s_{i}$ are illustrated in Figure 1.

Insert Figure 1: Disclosure function $\xi_{i}$ and disclosure time $t_{i}$ here.

We use the static payments (18) in the ascending mechanism, but only via the (conditioning) information available at the stopping time $\tau$. The individual exit times of the losing bidders, $\tau_{j} \leq \tau$, implicitly define the reported signal realizations $\widehat{s}_{j}$ via (19), namely

$$
W_{j}\left(\widehat{v}_{j}, \widehat{s}_{j}\right)=\tau_{j} .
$$

Thus, the winning bidder $i$ pays for all realizations of $s_{i}$ above the threshold $\underline{s_{i}}\left(\widehat{\mathbf{v}}, \widehat{\mathbf{s}}_{-i}\right)$, and we define the transfer function $P_{i}\left(\widehat{\mathbf{v}}, \widehat{\mathbf{s}}_{-i}\right)$ as

$$
P_{i}\left(\widehat{\mathbf{v}}, \widehat{\mathbf{s}}_{-i}\right) \triangleq \mathbb{E}\left[p_{i}\left(\widehat{\mathbf{v}}_{-i}, \widehat{\mathbf{s}}\right) \mid s_{i} \geq \underline{s_{i}}\left(\widehat{\mathbf{v}}, \widehat{\mathbf{s}}_{-i}\right)\right] .
$$


The winning bidder pays in expectations now as much as he would in the static mechanism with observable signals.

If we consider the allocation and payment rules, as encoded by (16) and (18), then it is apparent that all the decisions with respect to bidder $i$, whether they concern the disclosure of information or the allocation, only depend on the competing bidders in a very limited way; namely via the largest virtual utility among the competing bidders. Thus, to the extent that the other bidders are truthtelling, a sufficient statistic of the profile $\left(\mathbf{v}_{-i}, \mathbf{s}_{-i}\right)$ is the resulting maximal virtual utility

$$
w\left(\mathbf{v}_{-i}, \mathbf{s}_{-i}\right) \triangleq \max _{j \neq i}\left\{W_{j}\left(v_{j}, s_{j}\right), 0\right\}
$$

It follows that to verify the posterior incentive and participation constraints of bidder $i$, it is entirely sufficient to represent the competitors via a distribution of competing (maximal) virtual utilities $w$, which we denote by $G(w)$. For the remainder of this section, it will therefore be sufficient to consider a single agent competing against a virtual valuation $w$. In consequence we can drop the subscripts everywhere and rewrite the relevant notation, in particular (18) and (17):

$$
\underline{s}(\widehat{v}, w) \triangleq \min \{s \mid W(\widehat{v}, s) \geq \max \{w, 0\}\}
$$

and

$$
\underline{v}(s, w) \triangleq \min \{v \mid W(v, s) \geq \max \{w, 0\}\}
$$

Consequently, the transfer payment given by (18) can be written as

$$
p(s, w) \triangleq u(\underline{v}(s, w), s)
$$

where the transfer has a Vickrey property with respect to $v$ but not with respect to $s$.

Now, as $s$ is not observable in the ascending disclosure mechanism, if the bidder with a reported type $\widehat{v}$ wins against the virtual valuation of $w$, then his true signal $s$ has to be sufficiently high, namely $s \geq \underline{s}(\widehat{v}, w)$, and the transfer payment is formed by the conditional expectation

$$
P(\widehat{v}, w) \triangleq \mathbb{E}[p(s, w) \mid s \geq \underline{s}(\widehat{v}, w)]=\frac{1}{1-\underline{s}(\widehat{v}, w)} \int_{\underline{s}(\widehat{v}, w)}^{1} u(\underline{v}(s, w), s) d s,
$$

where here and in all future integral expressions, we use the property that $s$ is uniformly distributed on the unit interval. By the construction of the payment $P(\widehat{v}, w)$ in $(25)$, it follows that

$$
p(\underline{s}(\widehat{v}, w), w) \leq P(\widehat{v}, w)
$$


as well as

$$
u(\widehat{v}, \underline{s}(\widehat{v}, w))-P(\widehat{v}, w) \leq 0,
$$

where we note that by construction $\widehat{v}=\underline{v}(\underline{s}(\widehat{v}, w), w)$. For later use, we collect some properties of the threshold signal and the payment.

\section{Lemma 2 (Payment and Signal Threshold).}

1. If $v>v^{\prime}$, then $\underline{s}(v, w)<\underline{s}\left(v^{\prime}, w\right)$ for all $w$.

2. $p(s, w)$ is increasing in $s$ and $w$.

3. $P(v, w)$ is increasing in $w$ and decreasing in $v$.

Proof. (1.) By Lemma 1, the virtual valuation is strictly increasing in $v$ and $s$, and hence it follows that the signal thresholds $\underline{s}(\cdot, w)$ have to have the reverse ranking of $v$.

(2.) The transfer function $p(s, w)$ is given by $u(\underline{v}(s, w), s)$, see (24). By Lemma 1 , it follows that if $s$ is increasing, then $u(\underline{v}(s, w), s)$ is increasing as well. By Lemma $1, W(v, s)$ is strictly increasing in $v$ and $s$, and hence $\underline{v}(s, w)$ is increasing in $w$, and since $u(v, s)$ is increasing in $v$, the result follows.

(3.) For a given $v$, the transfer function $P(v, w)$, see (25), is defined as a conditional expectation over all signal realization $s$ above a threshold $\underline{s}(v, w)$. This threshold is increasing in $w$ by the

monotonicity of $W(v, s)$, see Lemma 1. But by the previous argument, (2.), $p(s, w)$ is increasing in both $s$ and $w$, and hence the conditional expectation over $p(s, w)$ is increasing in $w$. After all, an increase in $w$ raises the expectation, given that the function $p(s, w)$ is increasing in $s$ for a given $w$, but also the function $p(\cdot, w)$ is shifted upwards by a shift in $w$.

For a given $w$, the transfer function $P(v, w)$, is defined as a conditional expectation over all signal realization $s$ above a threshold $\underline{s}(v, w)$. This threshold is decreasing in $v$ by the monotonicity of $W(v, s)$, see Lemma 1. But by the previous argument, $(2),. p(s, w)$ is increasing in $s$, and hence the conditional expectation over $p(s, w)$ is decreasing in $v$.

\subsection{Posterior Implementation}

We now establish that the ascending disclosure mechanism leads to truthtelling with respect to $v$ and $s$. This will establish our main result:

\section{Proposition 2 (Posterior Implementation).}

The ascending disclosure mechanism satisfies the posterior incentive and participation constraints for all agents. The seller extracts as much revenue as in the revenue maximizing auction with observable signals. 
The proof proceeds in several steps. We show in Lemma 3 that if the bidder reports both his type and his signal truthfully, then he obtains the same allocation and expected utility as in the revenue maximizing mechanism of Eső and Szentes [10]. In Lemma 4 we show that if the bidder reports his type $v$ truthfully, then he will also report his signal $s$ truthfully, that is he will exit the process as soon as he learns his true signal $s$. Then, Lemma 5 establishes that if the bidder reports his signal $s$ truthfully, he will also report his type $v$ truthfully. The final step of the argument, presented in Lemma 6, shows that lying both with respect to the type and the signal is not profitable either.

\section{Lemma 3 (Revenue Equivalence).}

Given truthtelling of $(v, s)$, the allocation and the expected net utility is identical to the revenue maximizing mechanism with observable signals.

Proof. The equivalence follows directly from the stipulated behavior at (23) and the expected payment stipulated by (24). In the static mechanism a bidder with type $v$ obtains

$$
\int_{0}^{1}\left[\int_{0}^{\max \{0, W(v, s)\}}[u(v, s)-u(\underline{v}(s, w), s)] d G(w)\right] d s .
$$

In the present sequential procedure, the bidder with type $v$ receives

$$
\int_{0}^{1}\left[\int_{\underline{s}(v, w)}^{1}[u(v, s)-u(\underline{v}(s, w), s)]\right] d s d G(w) .
$$

The equivalence of (28) and (29) now follows after exchanging the order of integration.

We can now verify that every agent reports his information truthfully in equilibrium.

\section{Lemma 4 (Truthful Signal Report).}

Given truthtelling of type $v$, the bidder is truthtelling about signal s.

Proof. Suppose the sequential procedure reaches $w$ and $s>\underline{s}(v, w)$, then we assign the object to the bidder and ask him to pay

$$
P(v, w)=\frac{1}{1-\underline{s}(v, w)} \int_{\underline{s}(v, w)}^{1} u(\underline{v}(s, w), s) d s,
$$

and since he does not know the signal realization $s$ either, the expected net utility is

$$
\frac{1}{1-\underline{s}(v, w)} \int_{\underline{s}(v, w)}^{1}[u(v, s)-u(\underline{v}(s, w), s)] d s .
$$


But since the virtual utility is increasing in $s$, see Lemma 1, it follows that

$$
\frac{\partial \underline{v}(s, w)}{\partial s}<0
$$

and hence for all $s>\underline{s}(v, w)$,

$$
u(v, s)-u(\underline{v}(s, w), s)>0,
$$

since $v>\underline{v}(s, w)$, and thus the bidder expects a positive utility, and is staying in the auction.

On the other hand, suppose he were to learn that his true signal is $s=\underline{s}(v, w)$, then he would quit the auction immediately, because his expected utility if he were to win at some later point $w^{\prime} \geq w$ is given by:

$$
\begin{aligned}
& u(v, \underline{s}(v, w))-P\left(v, w^{\prime}\right) \leq u(v, \underline{s}(v, w))-P(v, w) \\
= & u(v, \underline{s}(v, w))-\int_{\underline{s}(v, w)}^{1} u(\underline{v}(s, w), s) \frac{d s}{1-\underline{s}(v, w)} \leq 0 .
\end{aligned}
$$

Now,

$$
u(v, \underline{s}(v, w))-u\left(\underline{v}\left(s^{\prime}, w\right), s^{\prime}\right)<0,
$$

since with $s^{\prime}>\underline{s}(v, w)$ and $v^{\prime}<v$ such that $u(v, \underline{s}(v, w))=u\left(v^{\prime}, s^{\prime}\right), W(v, \underline{s}(v, w))>W\left(v^{\prime}, s^{\prime}\right)$, by Lemma 1. But this means that $\underline{v}\left(s^{\prime}, w\right)>v^{\prime}$, and hence

$$
u(v, \underline{s}(v, w))-u\left(\underline{v}\left(s^{\prime}, w\right), s^{\prime}\right)<u(v, \underline{s}(v, w))-u\left(v^{\prime}, s^{\prime}\right)=0
$$

which completes the proof.

We are now in a position to verify that, conditional on reporting truthfully in the ascending auction, each bidder is also willing to report truthfully about his type $v$.

\section{Lemma 5 (Truthful Type Report).}

Given truthtelling of the signal s, the bidder is truthtelling about his type $v$.

Proof. Suppose for now that the bidder knows the value of $w$. Suppose also that the bidder misreports $\widehat{v} \neq v$ but continues to report his signal truthfully, that is he exits whenever his signal $s$ has been disclosed to him, i.e. $d(t, v, \widehat{v}, \xi(t, \widehat{v}, s))=0$ if and only if $\xi(t, \widehat{v}, s)>0$. Then, the agent will fail to win the object if $s<\underline{s}(\widehat{v}, w)$, which happens with probability $\underline{s}(\widehat{v}, w)$. Now, if $\underline{s}(\widehat{v}, w)=1$, then the proof is complete, since in this case this deviation yields a zero net payoff, 
and thus not profitable. Now suppose that $\underline{s}(\widehat{v}, w)<1$. The agent wins the auction if $s \geq \underline{s}(\widehat{v}, w)$ which happens with probability $1-\underline{s}(\widehat{v}, w)$, in which case he pays

$$
\frac{1}{1-\underline{s}(\widehat{v}, w)} \int_{\underline{s}(\widehat{v}, w)}^{1} u(\underline{v}(s, w), s) \mathrm{d} s .
$$

Therefore, his ex-ante expected payment is

$$
\int_{\underline{s}(\widehat{v}, w)}^{1} u(\underline{v}(s, w), s) \mathrm{d} s .
$$

His ex-ante gross utility derived from the object is

$$
\int_{0}^{\underline{s}(\widehat{v}, w)} 0 \mathrm{~d} s+\int_{\underline{s}(\widehat{v}, w)}^{1} u(v, s) \mathrm{d} s,
$$

so that his ex-ante net expected utility is

$$
\int_{\underline{s}(\widehat{v}, w)}^{1}[u(v, s)-u(\underline{v}(s, w), s)] \mathrm{d} s .
$$

Note that $u(v, s)-u(\underline{v}(s, w), s) \geq 0$ if and only if $v \geq \underline{v}(s, w)$, and in turn

$$
W(v, s) \geq W(\underline{v}(s, w), s)=W(v, \underline{s}(v, w))
$$

if and only if $\underline{s}(v, w) \leq s$. Therefore, the integral (31) is maximized if it is performed only on the interval on which the integrand is non-negative, which is by construction $[\underline{s}(v, w), 1]$. In other words, setting $\widehat{v}=v$ maximizes this integral. Since this holds for any $w$, it must also hold in expectation over all $w$.

For further analysis it is worth noting that the above proof establishes that reporting the true type is not just optimal in expectation over all possible competing virtual valuations $w$, but in fact for each realization of the virtual valuation $w$. The initial report $\hat{v}$ determines the speed by which the bidder runs through his signals. Now, for every $w$, an overreport is associated with a lower threshold for the critical signal $\underline{s}(v, w)$ by Lemma $2: \hat{v}>v \Leftrightarrow \underline{s}(\hat{v}, w)<\underline{s}(v, w)$. Similarly, for every $w$, an underreport is associated with a higher threshold for the critical signal $\underline{s}(v, w)$ by Lemma 2: $\hat{v}<v \Leftrightarrow \underline{s}(\hat{v}, w)>\underline{s}(v, w)$. Thus, if the bidder overreports his type, $\hat{v}>v$, the disclosure process ends earlier for the bidder, as the threshold for the disclosed signals $s$ is lower, $\underline{s}(\hat{v}, w)<\underline{s}(v, w)$. Thus, the bidder receives less private information, than if he were to report truthfully. By contrast, if the bidder underreports his type, $\hat{v}<v$, then the disclosure process ends 
later for the bidder, as the threshold for the disclosed signals is higher, $\underline{s}(\hat{v}, w)>\underline{s}(v, w)$. The initial reporting strategy of the bidder therefore influences the amount of private information that he will receive in the disclosure process. But the next result establishes that the advantage of increasing or decreasing the information is offset by less favorable transfer payments associated with underreports and overreports, respectively.

\section{Lemma 6 (Joint Deviations).}

The bidder cannot increase his utility by overreporting $\hat{v}>v$ or by underreporting $\hat{v}<v$.

Proof. We fix $w$ and consider the utility the bidder obtains as a function of his own signal $s$, if observed. We claim that for any misreport, the bidder obtains a lower utility for every $w$ than he would have obtained reporting his true type. We begin with overreporting $\hat{v}>v \Leftrightarrow \underline{s}(\hat{v}, w)<$ $\underline{s}(v, w)$. It is useful to consider two separate cases, and thus let

$$
V^{+} \triangleq\{v \in[0,1] \mid \widehat{v} \geq v \text { and the bidder wants the object upon learning that } s>\underline{s}(\widehat{v}, w)\}
$$

and conversely let

$$
V^{-} \triangleq\{v \in[0,1] \mid \widehat{v} \geq v \text { and the bidder rejects the object upon learning that } s>\underline{s}(\widehat{v}, w)\}
$$

Note that the agent prefers to receive the object upon learning that $s>\underline{s}(\widehat{v}, w)$ if and only if

$$
\mathbb{E}[u(v, s)-P(\widehat{v}, w) \mid s>\underline{s}(\widehat{v}, w)]=\frac{1}{1-\underline{s}(\widehat{v}, w)} \int_{\underline{s}(\widehat{v}, w)}^{1}[u(v, s)-u(\underline{v}(s, w), s)] \mathrm{d} s \geq 0 .
$$

Now suppose that $\widehat{v} \in V^{-}$and the agent learns that $s \leq \underline{s}(\widehat{v}, w)$, and hence $s \leq \underline{s}(\hat{v}, w)<\underline{s}(v, w)$, then it is optimal for the bidder to exit after $s$ has been revealed. After all, by overreporting $\hat{v}>v$, it follows that $u(\hat{v}, s)>u(v, s)$, for all $s$. But if $s \leq \underline{s}(\hat{v}, w)$, then by $(27), u(\hat{v}, s)-P(\hat{v}, w)<0$, and hence it follows that $u(v, s)-P(\hat{v}, w)<0$ as well, and thus exit is an optimal response, with the resulting zero net expected utility. If $\widehat{v} \in V^{-}$and $s>\underline{s}(\widehat{v}, w)$, then the agent will refrain from claiming the object by construction of (33), as well. Therefore, any deviation $\widehat{v} \in V^{-}$is unprofitable.

Now suppose that $\widehat{v} \in V^{+}$. Again, if $s \leq \underline{s}(\widehat{v}, w)$, then the agent will truthfully refrain from claiming the object. If $s>\underline{s}(\widehat{v}, w)$, then he will truthfully claim the object by construction. Therefore, if $\widehat{v} \in V^{+}$, then the bidder will optimally report his signal truthfully in the second stage for any realization of the signal. Now if $\widehat{v} \in V^{+} \backslash\{v\}$ would constitute a strictly profitable deviation, then we would have established a contradiction to Lemma 5, which established the optimality of truthtelling of the type, given thruthtelling of the signal. 
Next consider the case of underreporting: $\hat{v}<v \Leftrightarrow \underline{s}(\hat{v}, w)>\underline{s}(v, w)$. This implies that the bidder will learn more as compared to the case where he reported truthfully. If the signal $s$ is sufficiently small, then $s \leq \underline{s}(v, w)<\underline{s}(\hat{v}, w)$. Now, we observe that if the true signal had been $s=\underline{s}(v, w)$, then the bidder would not want to receive the object if offered at $P(v, w)$, since

$$
u(v, \underline{s}(v, w))-P(v, w)<0
$$

and by Lemma $2, P(\hat{v}, w)>P(v, w)$, and a fortiori would want to drop out of the auction. Suppose then that the true signal $s$ is sufficiently large, or $s>\underline{s}(v, w)$. Now, there must exist a signal $\tilde{s}$ with $\underline{s}(v, w)<\tilde{s} \leq \underline{s}(\hat{v}, w)$ such that the bidder buys the good (for the given $w$ ) if and only if his true signal is above $\tilde{s}$. Now, consider a type $\tilde{v}$ with $W(\tilde{v}, \tilde{s})=w$. By construction, the bidder who underreported $v$ to $\hat{v}$ obtains the object for the same set of signals as the truthful type $\tilde{v}$ would have. Note, however, that the payment of the type $v$ who underreports to $\hat{v}$ is larger than the payment of the $\tilde{v}$ type, again by Lemma 2. So, the utility of the bidder with type $v$, who underreports with regard to his type, and then behaves optimally with regard to his reported signal is smaller than if the bidder still underreported to $\tilde{v}$ and then reported his signal truthfully. But given Lemma 5, even the resulting net utility is smaller than the bidder would obtain if he were to report his type truthfully. Thus underreporting is not profitable either.

In the working paper, Bergemann and Wambach [3], we present an explicit solution with a single buyer and a utility function that is additive in the type $v$ and the signal $s$. The example illustrates the impact of the sequential information disclosure on the reporting incentives and the structure of payments and compares the transfers in the static disclosure environment of Eső and Szentes [10] with the transfers in the sequential disclosure environment.

\section{Discussion and Conclusion}

We extend the canonical single unit auction design to allow the seller to control the information that the bidder can receive about the object during the bidding contest. We exhibit a sequential disclosure mechanism associated with a sequential bidding mechanism which allowed the seller to extract almost the entire surplus of the allocation. The information rent of each bidder is restricted to the private information that each bidder was endowed with before entering the auction. The sequential disclosure process allowed us to assign the object in such a way as to maintain the posterior incentive and participation constraints of all the bidders. The disclosure mechanism allowed each bidder to obtain a sufficient amount of private information to find out whether his virtual valuation 
is larger or smaller than those of their competitors. Importantly, the winning bidder only learns the lower bound of his virtual utility, but never his exact valuation nor others' virtual valuations. This was achieved by informing the bidders in each round whether their valuations are below or above a given threshold.

We illustrate the main aspects of our ascending auction mechanism with a brief description of a common procurement practice, "request for quote" that shares many features with the present mechanisms. We end with a short discussion of the role of the representation of information for the disclosure process, and the interaction between the allocation problem and the disclosure process.

Request for Quote as a Sequential Disclosure and Bidding Process. The dynamic disclosure mechanism proposed has parallels to the commonly observed "request for quote" (RFQ) process in procurement auctions for items with many attributes, see Beil and Wein [1]. In this process, the buyer initially provides limited information about the object to the potential suppliers, which hand in a quote. On the basis of this first stage, selected suppliers are invited who obtain further, more detailed information about the product. In this procedure, the increased specification of the product goes in parallel with negotiations over prices and the number of potential suppliers is reduced over time until a winner is determined. Thus, in this sequential procedure suppliers learn more about the specification (and therefore about their costs) and only those able to compete further remain in the bidding process.

To illustrate, consider the following scenario. A firm that produces automotive and nonautomotive parts intends to procure a casting for one of its products. In general, many casting suppliers are eligible to supply. The RFQ process routinely proceeds in several steps in which the suppliers receive information about the part and are asked to quote an indicative price given this information. Suppliers that quote prices that are not competitive are eliminated from the process. In the first step of the procurement process, the purchasing firm may specify whether the part is intended for automotive production or not. If, as typical, quality requirements for automotive production are higher than for other productions, then suppliers that are not equipped for automotive production learn that a significant investment would be necessary to be even considered for the project. In a second step, the procuring firm announces general details of the project, e.g., required material. Most castings in automotive are either made from aluminum or steel and most castings suppliers are specializing in one of these materials. Suppose the part in question is made from aluminum. At this point suppliers that are specializing in steel would learn that they would need further investments to be able to supply the part. Next, technical blueprints are given to the 
suppliers, containing specific technical information. Suppliers learn whether the part in question is rather complex with many cavities or rather simple with just one cavity and thus whether the machines that they already own are sufficient to produce the required complexity. Next, the suppliers are given further detailed requirements, e.g. exact quality requirements like "ppm" (parts per million, a measure of the error rate of a part). At this point suppliers learn how much man-power is required to set-up and supervise the quality of production of the product and whether they have to hire more staff. The remaining suppliers can then improve their estimates of the cost of production and determine their final bid.

The technology structure and the information process in this example can be formalized as follows. The different characteristics of the object are given by a vector of dimension $K, x=$ $\left(x_{1}, \ldots, x_{K}\right)$. Each characteristic $k$ can take one of two values, $x_{k} \in\{0,1\}$. The different characteristics are ordered in importance, as described in the example, e.g. $x_{1}$ specifies whether the part is intended for automotive parts or not, $x_{2}$ specifies that the required material is aluminium and so on. The buyer reveals the vector of characteristics in sequential order.

The technology of the potential supplier $i$ can also be described by a vector of dimension $K$, $y_{i}=\left(y_{i 1}, \ldots, y_{i K}\right)$, where each entry $y_{i k} \in\{0,1\}$. The cost function of the supplier is given by the nested technology requirement and can be defined as a function of the length $z_{i}$ of the chain of consecutive matches of characteristics $x_{i}$ and preferences $y_{i k}$ :

$$
z_{i}=\max _{k}\left\{0 \leq k \leq K \mid y_{i l}=x_{l}, \forall l \leq k\right\}
$$

In particular, let $c_{i}\left(x, y_{i}\right)=1-z_{i} / K$. As long as the technology fits with the characteristics of the object, the supplier does not need to incur additional (and prohibitive) cost in production facilities to meet the requirements of the buyer. Thus, the failure to have a matching feature in step $k$ gives the final cost of production, and a success to match the current feature allows the bidder to maintain a positive expectation of the true cost of production.

Disclosing Signal $s_{i}$ versus Value $V_{i}$. In the present analysis, we restricted the seller to disclose information contingent on $s_{i}$ rather than $V_{i}$. We mentioned earlier that Li and Shi [18] recently showed that by allowing the disclosure policy to be contingent on $V_{i}$ rather than $s_{i}$, the seller can sometimes increase his expected revenue. As $V_{i}$ contains more information (about $V_{i}$ ) than $s_{i}$, the seller has a better instrument, and thus it is not entirely surprising that the revenue may improve. Li and Shi [18] consider the allocation problem of a single item to a single buyer. By means of an informative example, their Example 4, they show that partial disclosure in the form 
of a binary partition characterized by single threshold can constitute the optimal static disclosure policy. The threshold policy supports the sale of the object above the threshold $\underline{V}$ and no sale below the threshold $\underline{V}$. Interestingly, this static threshold policy is in fact very similar to the information that the bidders receive at every point in time in our sequential mechanism. Here, the threshold is increased in every round and thus the losing bidders learn that their valuations are below the threshold at the moment at which they exit the process, but crucially the winning bidder receives only partial information about his true value. We therefore suspect that if we were to compare the static and sequential disclosure policies based on $V_{i}$ rather than $s_{i}$, that the participation constraints of the bidders would similarly be strengthened by a sequential disclosure mechanism. In fact, their Example 4 has property that a sequential implementation with a single transaction payment and no participation payment constitutes an optimal mechanism. ${ }^{9}$

The Limits of Sequential Disclosure. The sequential disclosure mechanism modifies the disclosure of information in two important aspects relative to the complete disclosure of information in the static mechanism of Eső and Szentes [10]. It discloses the information (i) partially and (ii) sequentially. Crucially, the information remains only partially disclosed at the end of the mechanism. To wit, in the auction, the revenue-maximizing allocation can be realized with limited knowledge of the true value of the winning bidder. All that is needed is the information that the winning bidder has a value higher than all the losing bidders (or in the case of single buyer that his valuation is above the cost of the seller.) Thus, the sequential disclosure mechanism releases all the information that is necessary for the assignment of the object, but not more, and hence supports the partial disclosure of information. This also suggests the limits of a sequential disclosure mechanism. If the allocation problem would be nonlinear - rather than zero or one - as in the classic quantity or quality discrimination problems, then we would conjecture that the coarse information offered by the sequential disclosure would not be sufficient anymore to attain the revenue maximizing allocation.

\footnotetext{
${ }^{9}$ We thank Xianwen Shi for suggesting the sequential implementation of their static disclosure mechanism.
} 


\section{References}

[1] Beil, D., and L. Wein (2003): "An Inverse-Optimization-Based Auction Mechanism to Support a Multiattribute RFQ Process," Management Science, 49, 1529-1545.

[2] Bergemann, D., and M. Pesendorfer (2007): "Information Structures in Optimal Auctions," Journal of Economic Theory, 137, 580-609.

[3] Bergemann, D., and A. Wambach (2013): "Sequential Information Disclosure in Auctions," Discussion Paper 1900, Cowles Foundation for Research in Economics.

[4] Blumenthal, M. (1988): "Auctions with Constrained Information: Blind Bidding for Motion Pictures," Review of Economics and Statistics, 70, 191-198.

[5] Boone, J., and J. Goeree (2009): "Optimal Privatization under Asymmetric Information," Economic Journal.

[6] Calzolari, G., and A. Pavan (2006a): "Monopoly with Resale," RAND Journal of Economics, 37, 362-375.

[7] (2006b): "On the Optimality of Privacy in Sequential Contracting," Journal of Economic Theory, 130, 168-204.

[8] Cassady, R. (1967): Auctions and Auctioneering. University of California Press.

[9] Demsetz, H. (1968): "Why Regulate Utilities?," Journal of Law and Economics, 11, 55-65.

[10] Eső, P., And B. Szentes (2007): "Optimal Information Disclosure in Auctions," Review of Economic Studies, 74, 705-731.

[11] Gershrov, A. (2002): "Optimal Auctions and Endogenous Information Structure," Discussion paper, Hebrew University, Jerusalem.

[12] (2009): "Optimal Auctions and Information Disclosure," Review of Economic Design, $13,335-344$.

[13] Green, J., and J. Laffont (1987): "Posterior Implementability in a Two Person Decision Problem," Econometrica, 55, 69-94. 
[14] Kamenica, E., And M. Gentzkow (2011): "Bayesian Persuasion," American Economic Review, 101, 2590-2615.

[15] Kavajecz, K., and D. Keim (2005): "Packaging Liquidity: Blind Auctions and Transaction Cost Efficiencies," Journal of Financial and Quantitative Analysis, 40, 465-492.

[16] Kenney, R., And B. Klein (1983): "The Economics of Block Booking," Journal of Law and Economics, 26, 497-540.

[17] Lebrun, B. (2010): "First-price auctions with Resale and with Outcomes Robust to Bid Disclosure," RAND Journal of Economics, 41, 165-178.

[18] Li, H., And X. Shi (2013): "Discriminatory Information Disclosure," Discussion paper, University of British Columbia and University of Toronto.

[19] Loeb, M., And W. Magat (1979): "A Decentralized Method of Utility Regulation," Journal of Law and Economics, 22, 399-404.

[20] Pavan, A., I. Segal, and J. Toikka (2014): "Dynamic Mechanism Design: A Myersonian Approach," Econometrica, 82, 601-653.

[21] YE, L. (2007): "Indicative Bidding and a Theory of Two-Stage Auctions," Games and Economic Behavior, 58, 181-207. 


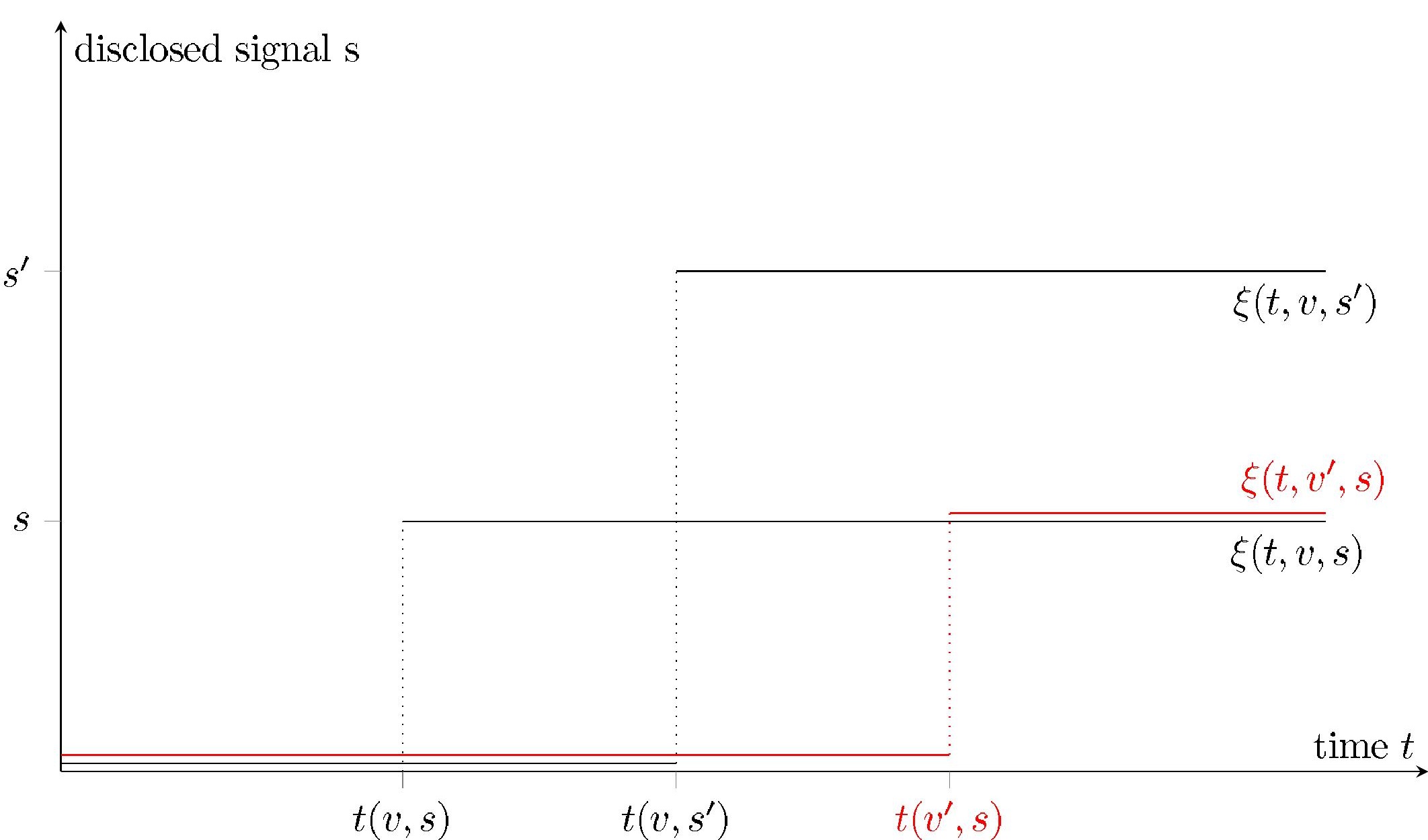

disclosed signal s

$$
\begin{array}{ccc}
t(v, s) & t\left(v, s^{\prime}\right) & t\left(v^{\prime}, s\right)
\end{array}
$$

